\title{
Examination of Location Based Services and User Preferences
}

\author{
C. Karpagam, S. Gomathi
}

\begin{abstract}
Recent developments on mobile location information have driven efforts to mine user patterns of interest. Even start-up companies survey user interests to enrich their business. All medium and large organizations are paying attention to collect and store location data. With the support of unlimited computing power and memory of mobile phones we can apply proficient Deep Learning algorithms to determine an optimal solution for user interests. In this article, we aim to complete an overall survey on evolution of Location Based Services and the improvements in recent trends. We have categorized the evolution period in to three divisions covering from the year 2000 to till date.
\end{abstract}

Keywords : challenges, location based services, recent trends, user preferences.

\section{INTRODUCTION}

$\mathrm{T}$ he tremendous growth in number of Smartphone users worldwide has crossed three billion and is expected to further increase by several hundred million in the next few years [1]. The changing behavior clearly denotes that consumers are now considering Smartphone's a necessary expense rather than a discretionary one. Mobile phone has become more than just a communication tool, it's now an entertainment device, computer and camera all rolled into one.

Smartphone penetration and low data cost have increased digital access among users. The increasing usage of Smartphone also pays way to the exponential growth of mobile applications. According to a recent survey, location based mobile apps are widely accessed more in number among users. Popular location based service applications are device tracking app, navigation, social media, marketing, emergency, sports, targeted advertisements, entertainment, games and augmented reality. Many more applications are up trending the market. Few collections were shown in Fig. 1.

Geofencing, geotagging, and location-based advertisements have all risen up to next level of advancement. More number of mobile applications is trending with LBS data. Apart from entertainment and games it assists the user to find valuable information from the hidden data. For example, if the user plans to open a new book store at a prime location in the city. The application can provide vital information such as, percentage of book readers at the destined location, existing number of book stores in the intended location and even the type of books preferred by the readers. This analysis helps the new business makers to make critical decision. The prominent usage of location data finds more interesting and expensive information. These strategies were also focused by retailers, restaurants and marketing initiatives to improve their business.

This provides an opportunity to government and corporate sectors to establish and improve their application in a better way. This also provides a path to various researchers to work on improving the algorithms and analysis report of a system vividly. Along with the location information we also concentrated in personalizing the content for different users. In lieu of generating the same result, the user will obtain their favorite results.

This paper periodically reviews the techniques applied to Location services at different stages to improve the performance. We aim to project the content from the year 2000 to till date. Classification is based on method of approach, pitfalls in existing, corrective measures etc.

\section{LOCATION BASED SERVICES (LBS)} (2000 - 2005)

The improvements in sensor and wireless communication technology have paved way to development of Location Based Services. This time period plays a major role in the mobile transformation from simple talk based services to incorporate multimedia features like sending pictures, audio, animation, flash messages and video file. Public Wi-Fi networks began to provide location-based information and services to intended users. LBS have started hitting the market during this period. Initially, the primary objective of LBS is to provide travel related services to tourists [10], "Buddy Services" that informs friends when they are physically close to each other [11]. Later it enters into business, marketing, entertainment, emergency, safety, medical and other services which we are currently on [13].

The primary discussion on the articles is method used to locate the user. The user should include a GPS (Global Positioning System) module to determine the position accurately [11], [14].Another main concern about the advancing technology is privacy issues. Many research articles were published on privacy principles, threats and data protection of user's

Revised Manuscript Received on October 31, 2019.

* Correspondence Author

C. Karpagam*, Department of Computer Science, Dr. N. G. P. Arts and Science College, Coimbatore, India. Email: karpagamchandran@yahoo.in

Dr. S. Gomathi, Department of Computer Applications, Sri Ramakrishna College of Arts and Science, Coimbatore, India. Email: gomathi.s@srcas.ac.in 


\section{Examination of Location Based Services and User Preferences}

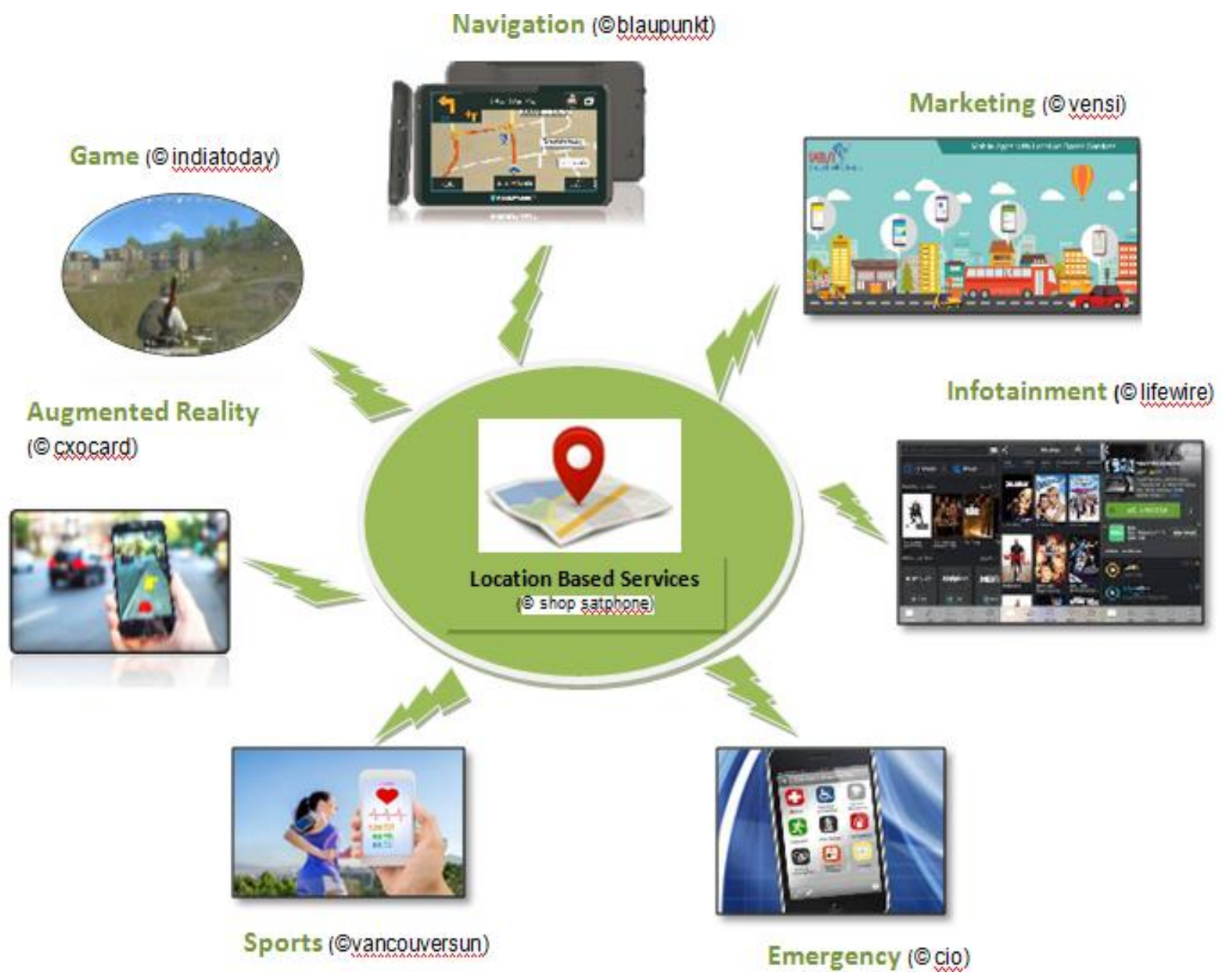

Fig. 1. Different application domains of LBS

personal information [7] - [9], [11], [12]. Adaptive-Interval Cloaking Algorithm is used to maintain high degree of anonymity among different users.

Other serious issues focused are response time, improving the accuracy of system, to reduce communication costs between server and client. Cost reduction techniques were imposed to decrease the number of messages form client to server and vice-versa [8]. Researchers also focused on challenges like limited capacity of handheld devices, expensive operating costs, limited bandwidth, user interaction via small screens, limited battery power, integration of heterogeneous services and fast access to internet service providers etc., the issue covered in this time period largely focussed on the capacity of devices that handles location data.

\section{CONTEXT AWARE LOCATION INFORMATION SERVICES (2006 - 2010)}

The massive propagation and widespread adoption of mobile phone and location data allows the service providers to exploit customer information and analyse the acquired user data. During this period pinpointing user location has been difficult due to its inherent dynamism and unpredictability of customer's location. Soon researchers focus on developing new technologies to embed location information in lightweight mobile devices and terminals. This led to the next level of advancement in LBS (i.e.)
Context-aware location information which, focus on location dependent data.

Context Aware information model provides location information dynamically based on environment and user needs. Relying only on location information sometimes may lead to irrelevant results. For example, consider the offers provided by telecom companies for mobile recharge. They were not same to all the subscribers. It varies according to particular day, time of access, location of the subscriber, type of subscriber etc. These kinds of features attract the customers to continue with the same network. This technique acts as one kind of business strategy to seek attention of users. Therefore for effective results LBS should provide information not only based on location but also focus on context information of the requester [2]. Another example is location based advertising that provides richer and more meaningful information based on the user context [3]. The user might be happy to receive a surprise discount offer from his/her favorite restaurant during an evening drive.

Context aware systems emerge in the market, the improvements made is to give more optimal solution to the existing location based data. Such systems can respond intelligently to their physical world changes acquired by using current scenario and according to the information about the computational environment and user's needs [4]. 
Context aware systems is to take smart decision about, when, where and what service to be provided to the user? Returned result would be more efficient, dynamic and intelligent decision the user might make use of. The different types of context factors are environment, time, and purpose of use, history of user data, mobility of the user, direction, speed of travel, and the properties of mobile phone.

Product recommendation applications were developed during this decade that automatically integrates user's shopping needs with location-dependent offers [15]. An approach is formulated by extending the context value using ontology [16], [17]. Ontology based approach is used for content recommendation towards context-aware e-learning.

Collaborative filtering is a popular approach to determine user interest area by obtaining voting from similar users. The system generates correlation, a statistical relationship between users with similar interests. But this fails due to the time complexity of algorithms by processing huge datasets in real time scenario. The next improvement was made to determine similarity among voted items instead of concentrating the similarity among users.

Compared with Collaborative filtering, Bayesian Networks (BN) Recommendation system provides high accuracy of prediction. The BN model is categorized into three modules. First module aims to obtain user information from mobile device such as, time, day, context information, weather and season. Second module applies Expectation Maximization (EM) algorithm to determine user preference based on restaurant dataset such as restaurant type, price, etc. The third module infers the highest probability data by proposing a map based recommendation system. The BN model provides high accuracy of data and the final results embedded in a mini map, which is flexible to view in small screen devices [18].

Limitation found in this period of LBS is, it provides the same result for all the users in the particular context. But user preferences differ from one person to other. The next advancement concentrates on individual user needs and priorities.

\section{PERSONALIZED LOCATION BASED SERVCICE RECOMMENDATION (LBSR)} (2011 - TILL DATE)

\section{A. Point of Interest (POI) calculation based on user data}

Obtained context aware information is common to all users. But the user expectation is not same all the time, different users have different opinions. During this period, context-aware systems concentrate on the requirements of both environment and the user preferences. Earlier context-aware architectures tend to treat the need of no-human intervention by static design artefacts. Much flexible and adaptive architecture is perceived when user data is combined [19].

Addressing the influence factors of user background details such as cognitive abilities, personality differences and psychological differences vary from one user to another [20]. But determining the user data is another complex task because; there might be only few historical records of the target user around the current location. Context aware systems often suffer from the limited quantity of users' check-in data.
LBSNs feature a huge number of locations with a large variety, but in practice users visit a very limited number of locations, therefore the user check-in data is extremely sparse [21]. Another interesting aspect includes identifying travel route to commuters. The algorithms used to compute travel information requires more computing power and time to retrieve results on time. Due to the high latency of algorithms and different type of user driving mode the exact result is highly questionable. Therefore many research articles were published to assist commuters. For example, working people often start to office in the morning and reach the same destination in the evening is quite common. This round-trip travel process is optimized by providing accurate travel information before the traveller starts his journey. O. Mazhelis suggested a personal route assistance system that predicts accurate travel information in advance [29].

The system is verified with a sample dataset of 20 drivers and their car movements for several months. The data set maintains the trajectory information of each driver and their location information in sequence of time stamps. Now the algorithm takes this history of user routes as input and predicts accurate travel information. Other parameters considered are external factors such as weather information, traffic data and time of the day during travel. The algorithm aims to find the similarity between the current route and the referenced route stored in the data set. The prediction accuracy is evaluated in three levels best, average and worst ranks.

\section{B. POI calculation based on Location Based Social Networks (LBSNs)}

Here the next focus is to address the data scarcity problem on user dependent information. Due to insufficient data on individual user mobile-history, an efficient outcome cannot be expected. Therefore the next step of precedence made is to collect the information from similar type of users in social network.

Geo-Sparse Additive Generative Model considers both user personal interests and the preference of the crowd in the target region. This particular set of data can be obtained through social networks, friend circle, and people with similar interests [22], [23]. LBSN paves way to recommend new venues and events on location detail to similar users. Geographic SAGE Model combines user interest data along with the influencing crowd in the locality. An additional parameter is set to differentiate the user from native to tourist, which results in different set of outputs. User activity profile is considered to be the input data. The model adopts to choose a location by matching the query with user personal interests. The computed results provide $\mathrm{k}$ recommendations to users by considering their own interests and preferences. Significant performance accuracy is denoted in the evaluation of training and test data sets.

\section{Spatial Keyword Query Processing}

To improve the results further Spatial Keyword Query Processing (SKQP) is implemented where it takes a user location and user-supplied keywords as arguments and returns spatial objects related to user typed keywords [24], [25]. 


\section{Examination of Location Based Services and User Preferences}

For example, consider a user looking for a hotel stay with a swimming pool and bar attached. The keywords are applied to retrieve the specific spatial object.

SKQP examines the distinct keywords entered by the user and construct a tree structure to determine the weighted average on spatial objects. The approximation algorithm finds user relevant search results.

\section{RECENT FOCUS ON DEEP LEARNING APPROACH}

Predicting the outcome of results is quite common. People often predict results on weather patterns, political events, sports outcomes, and more. Prediction is done using simple intuition, expert opinions or by applying past results to compare with traditional statistical techniques.

Deep Learning is a form of machine learning that teaches computers to complete their task efficiently. Deep learning is able to learn from unstructured and unlabelled data. Used as a key technology behind automated vehicles that helps the machine to recognize traffic signs, signals and direction boards. It also acts as a responding device to control voice input in devices like phones, tablets, TVs, etc. Deep learning is getting lots of importance in various dimension for a good reason and also it achieves results that were not possible before.

The deep learning method Recurrent Neural Networks (RNNs) has been considered for predicting the outcomes of football matches. The final result is predicted with the help of previous recorded matches held at 63 different countries [5]. A deep learning based approach is used to classify travellers' behaviours depends on various characteristics, such time, day, mode of travel, previous visits, purpose of journey etc,. [6]. Based on the above categories the author classify the results into 5 clusters: shopping activities of working and non-working people, commuters etc., The final results are $95 \%$ in accuracy. This clearly shows that deep learning achieves higher accuracy at different levels than ever before.

A new approach is applied by DongWang et. al. [6] to estimate the travel time based on DeepTTE (Deep learning framework for Travel time Estimation). The model present the result based on three components: attribute, spatio-temporal and multi-task learning component. Extensive results are obtained when experimented with real data sets. Similarly a deep learning approach can be applied to extract the user data and analyze his/her preference to predict their choices and interest in location based services.

Popular filtering techniques and collaborative models were suggested to determine user preference results. Matrix Factorisation (MF), Neural Matrix Factorization, Recurrent Neural Network based approaches are popular Collaborative Filtering (CF) techniques suggested to users based on an assumption that similar users are likely to visit similar venues. The proposed model generates a ranked list of recommended venues based on history of feedback [26], [27]. Experimental results are focussing on ranking user preference data more effectively [28]. Privacy and threat of user data is another aspect of ongoing research in all stages of development. Deep learning mainly focuses on to provide more intelligent and accurate result on user data.

\section{CONCLUSION}

Due to the increase in adoption of mobile devices and location aware system, more intelligent systems are developed and this continues to grow. Therefore in this article, we initially reviewed the start up demands for location data. The second period of development reviewed on the improvements made to give positive shape and future awareness to location services. The third cycle of the development period shows tremendous improvement in technologies and different sectors of user targets. We believe that the survey on location aware systems stated in this paper covers the most essential and fundamental content in the evolution of LBS.

\section{REFERENCES}

1. https://www.statista.com/statistics/330695/number-of-smartphone-user s-worldwide/

2. Haosheng Huang, Georg Gartner, Jukka M. Krisp, Martin Raubal \& Nico Van de Weghe, "Location based services: ongoing evolution and research agenda", Journal of Location Based Services, 12:2, 63-93,DOI: 10.1080/17489725.2018.1508763

3. Toch, E., Lerner, B., Ben-Zion, E. et al. Knowl Inf Syst (2019) 58: 501 https://doi.org/10.1007/s10115-018-1186-x

4. Mcheick Hamid. (2014). "Modeling context aware features for pervasive computing". Procedia Computer Science, 37, p. 135-142.

5. Pettersson, D., and R. Nyquist. "Football Match Prediction using Deep Learning Recurrent Neural Network Applications." Master's Thesis EX031/2017. Department of Electrical Engineering Chalmers University of Technology. Typeset in L ATEX. Gothenburg, Sweden (2017).

6. Wang, Shenhao \& Zhao, Jinhua. (2018). Using Deep Neural Network to Analyze Travel Mode Choice With Interpretable Economic Information: An Empirical Example.

7. Marco Gruteser, Dirk Grunwald, Anonymous Usage of Location-Based Services Through Spatial and Temporal Cloaking, Proceedings of the 1st international conference on Mobile systems, applications and services, p.31-42, May 05-08, 2003, San Francisco, California

8. Kido, Hidetoshi \& Yanagisawa, Y \& Satoh, Tetsuji. (2005). An Anonymous Communication Technique using Dummies for Location-based Services. Proceedings - International Conference on $\begin{array}{lllll}\text { Pervasive Services, ICPS } & \text { '05. 88-97. } & 88- & 97\end{array}$ 10.1109/PERSER.2005.1506394.

9. Barkhuus, Louise \& Dey, Anind. (2003). Location-Based Services for Mobile Telephony: a Study of Users' Privacy Concerns.. 2003.

10. Schmidt-Belz, Barbara \& Nick, Achim \& Poslad, Stefan \& Zipf, Alexander. (2003). Personalized and location-based mobile tourism services.

11. Myles, G \& Friday, Adrian \& Davies, N. (2003). Preserving Privacy in Environments with Location-Based Applications. Pervasive Computing, IEEE. 2. 56- 64. 10.1109/MPRV.2003.1186726.

12. Zhong, Sheng et al. "Privacy-Preserving Location-based Services for Mobile Users in Wireless Networks." (2004).

13. Steinfield, Charles. (2003). The Development of Location Based Services in Mobile Commerce 1. 10.1007/978-3-662-11659-3_10.

14. Kaasinen, Eija. "User needs for location-aware mobile services.” Personal and Ubiquitous Computing 7 (2003): 70-79.

15. Yang, Wan-Shiou \& Cheng, Hung-Chi \& Dia, Jia-Ben. (2008). A Location-Aware Recommender System for Mobile Shopping Environments. Expert Systems with Applications. 34. 437-445. 10.1016/j.eswa.2006.09.033.

16. Xiao, Hua \& Zou, Ying \& Ng, Joanna \& Nigul, Leho. (2010). An Approach for Context-Aware Service Discovery and Recommendation. 163-170. 10.1109/ICWS.2010.95.

17. Yu, Zhiwen \& Nakamura, Yuichi \& Jang, Seiie \& Kajita, Shoji \& Mase, Kenji. (2007). Ontology-Based Semantic Recommendation for Context-Aware E-Learning. 898-907. 10.1007/978-3-540-73549-6_88.

18. Park, Moon-Hee \& Hong, Jin-Hyuk \& Cho, Sung-Bae. (2007) Location-Based Recommendation System Using Bayesian User's Preference Model in Mobile Devices. Comput. Sci.. 1130-1139. 10.1007/978-3-540-73549-6_110. 
19. Mcheick, Hamid. (2014). Modeling Context Aware Features for Pervasive Computing. Procedia Computer Science. 37. 10.1016/j.procs.2014.08.022.

20. O. Roider, C. Rudloff, M. Ray, "An Individual Travel Information System for Optimizing Mode and Route Choice bahaviour of Commuters", in Proc. Social and Behavioral Sciences 48, 2012, pp. 1948-1957

21. Aliannejadi, Mohammad \& Rafailidis, Dimitris \& Crestani, Fabio. (2018). A Collaborative Ranking Model with Multiple Location-based Similarities for Venue Suggestion. 10.1145/3234944.3234945.

22. Yin, Hongzhi \& Sun, Yizhou \& Cui, Bin \& Hu, Zhiting \& Chen, Ling. (2013). LCARS: a location-content-aware recommender system. 221-229. 10.1145/2487575.2487608.

23. Wang, Weiqing \& Yin, Hongzhi \& Chen, Ling \& Sun, Yizhou \& Sadiq, Shazia \& Zhou, Xiaofang. (2015). Geo-SAGE: A Geographical Sparse Additive Generative Model for Spatial Item Recommendation.

24. Hariharan, Ramaswamy \& Hore, Bijit \& Li, Chen \& Mehrotra, Sharad. (2007). Processing Spatial-Keyword (SK) Queries in Geographic Information Retrieval (GIR) Systems. Proceedings of the International Conference on Scientific and Statistical Database Management, SSDBM. 16-16. 10.1109/SSDBM.2007.22.

25. Chen, Lisi \& Cong, Gao \& Jensen, Christian \& Wu, Dingming. (2013). Spatial keyword query processing: An experimental evaluation. Proceedings of the VLDB Endowment. 6. 217-228. $10.14778 / 2535569.2448955$.

26. Manotumruksa, Jarana \& Macdonald, Craig \& Ounis, Iadh. (2017). A Deep Recurrent Collaborative Filtering Framework for Venue Recommendation. 1429-1438. 10.1145/3132847.3133036.

27. Aliannejadi, Mohammad \& Rafailidis, Dimitris \& Crestani, Fabio. (2018). A Collaborative Ranking Model with Multiple Location-based Similarities for Venue Suggestion. 10.1145/3234944.3234945.

28. Aliannejadi, Mohammad \& Crestani, Fabio. (2018). Venue Suggestion Using Social-Centric Scores.

29. Mazhelis, Oleksiy \& Žliobaitè, Indrè \& Pechenizkiy, Mykola. (2011) Context-Aware Personal Route Recognition. Performance Evaluation PE. 221-235. 10.1007/978-3-642-24477-3_19.

\section{AUTHORS PROFILE}

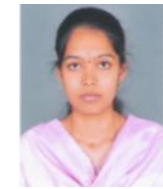

C. Karpagam owns Masters Degree in Computer Applications. She is currently an Assistant Professor at Dr. N. G. P. Arts and Science College, Coimbatore, India. Her research interests include spatial data and location information. She is a member in Association for Computing Machinery (ACM) and Indian Society for Technical Education (ISTE).

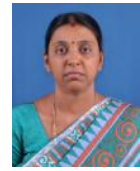

Dr. S. Gomathi @ Rohini owns 13 years of academic experience and 3 years of industrial experience. Image processing and analytics are her fields of interest. She has more than 20 publications in her credit. She is currently serving as Associate Professor Computer Science in Sri Ramakrishna College of Arts \& Science, Coimbatore. She is supervising a few Ph.D./M.Phil. projects. She is a member in IAPA and IEDRC. She has organised a few funded workshops. 